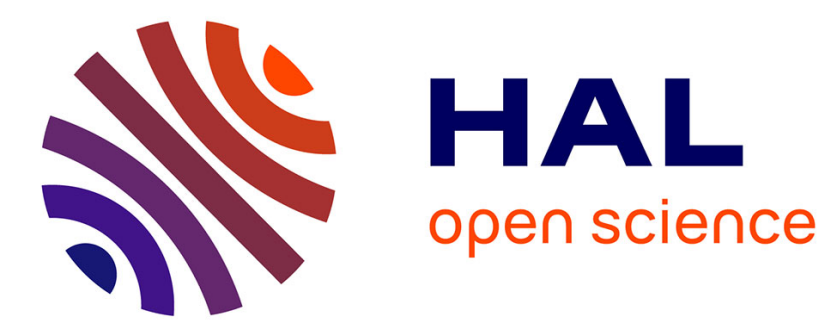

\title{
Theoretical investigation of the platinum substrate influence on BaTiO 3 thin films polarisation
}

Pierre-Marie Deleuze, Agnes Mahmoud, Bruno Domenichini, Céline Dupont

\section{To cite this version:}

Pierre-Marie Deleuze, Agnes Mahmoud, Bruno Domenichini, Céline Dupont. Theoretical investigation of the platinum substrate influence on $\mathrm{BaTiO} 3$ thin films polarisation. Physical Chemistry Chemical Physics, 2019, 21, pp.4367-4374. 10.1039/C8CP07022A . hal-03032729

\section{HAL Id: hal-03032729 \\ https://hal.science/hal-03032729}

Submitted on 1 Dec 2020

HAL is a multi-disciplinary open access archive for the deposit and dissemination of scientific research documents, whether they are published or not. The documents may come from teaching and research institutions in France or abroad, or from public or private research centers.
L'archive ouverte pluridisciplinaire HAL, est destinée au dépôt et à la diffusion de documents scientifiques de niveau recherche, publiés ou non, émanant des établissements d'enseignement et de recherche français ou étrangers, des laboratoires publics ou privés. 


\title{
Journal Name
}

\section{ARTICLE TYPE}

Cite this: DOI: $10.1039 / x x x x x x x x x x$

\section{Theoretical investigation of the platinum substrate in- fluence on $\mathrm{BaTiO}_{3}$ thin films polarisation.}

\author{
Pierre-Marie Deleuze,${ }^{a}$ Agnes Mahmoud,${ }^{a}$ Bruno Domenichini, ${ }^{a}$ and Céline Dupont ${ }^{* a}$
}

Received Date

Accepted Date

DOI: 10.1039/xxxxxxxxxx

www.rsc.org/journalname

\begin{abstract}
Density functional theory calculations are performed to study the out-of-plane polarisation in $\mathrm{BaTiO}_{3}$ (BTO) thin films epitaxially grown on platinum. Prior to any polarisation calculation, the stability of the $\mathrm{Pt}(001) / \mathrm{BaTiO}_{3}(001)$ structure is thoroughly discussed. In particular, the nature of the Pt/BTO and BTO/vacuum interfaces is characterised. The growth of BTO is shown to start with a $\mathrm{TiO}_{2}$ layer while the nature of the surface termination does not broadly modify the stability. Therefore both upper terminations are considered when describing the ferroelectric behaviour in Pt/BTO interfaces. The geometric and electronic effects of the substrate on the polarisation are investigated. To isolate the electronic influence of platinum, the out-of-plane polarisation in Pt/BTO systems is compared to the one in isolated BTO slabs constrained to the same lattice mismatch induced by the epitaxial growth on platinum. The ferroelectric phase is favoured as soon as the thickness is larger than $23 \AA$, both for isolated and deposited BTO, for the smallest width. The Pt substrate will modify the size of polarisation domains, while an upper $\mathrm{BaO}$ layer through the use of asymmetric $\left[\mathrm{TiO}_{2} / \mathrm{BaO}\right]$ systems, will induce an increase of the polarisation. One could take advantage of this experimentally.
\end{abstract}

\section{Introduction}

Efficient use of renewable energies represent one of the main challenges of the last decades. Among them, solar energy can be used to produce hydrogen through water photo electrolysis. To do so, metal oxide could constitute a wide class of suitable materials, however their efficiency is often very poor. Several reasons can be invoked, like the wrong conduction band alignment of usual metal oxide catalysts as well as the decrease of the electron-hole recombination rate ${ }^{1+4}$. While an external bias can be used to align bands properly, the most profitable option is to find an internal solution. From this point of view, the ability of ferroelectric materials to exhibit an intrinsic polarisation and thus a permanent internal electric field is a great opportunity. As a consequence, ferroelectric materials have set off considerable attention in the past years for photocatalytic reactions $s^{5 \cdot 7}$ or photovoltaic devices 8 .10. Moreover, several studies have showed that the dipolar field can influence charge carrier transport leading to a spatial localisation of the electron and hole half reactions ${ }^{11-14}$. Hence, it is of primary importance to study the factors influencing the polarisation in order to take advantage of it.

Among possible ferroelectric oxides, $\mathrm{BaTiO}_{3}$ (BTO) has been extensively studied over the last decades as it stands for an archety-

\footnotetext{
${ }^{a}$ Address, Laboratoire Interdisciplinaire Carnot de Bourgogne (ICB), UMR 6303 CNRS, Université de Bourgogne Franche Comté, BP 47870, 21078 Dijon Cedex, France; Email: celine.dupont@u-bourgogne.fr
}

pal ferroelectric perovskite. At high temperature, it shows a paraelectric cubic structure where $\mathrm{Ba}^{2+}$ ions are located at the corners, $\mathrm{O}^{2-}$ ions at the center of the faces and a $\mathrm{Ti}^{4+}$ ion at the body center of the cell. Below the Curie temperature (around $120^{\circ} \mathrm{C}$ ), BTO undergoes a transition to a tetragonal ferroelectric phase which exhibits a spontaneous polarisation along the [001] direction. The cubic phase of $\mathrm{BTO}(001)$ has already been experimentally described ${ }^{[15}$ as well as its tetragonal phase ${ }^{16-18}$. Numerous studies $\sqrt{19,26}$ have been devoted to $\mathrm{BaTiO}_{3}$ involved in capacitors, namely BTO sandwiched between two electrodes. All these studies have allowed the characterisation of different properties of BTO, such like the critical thickness required for polarisation. On the contrary, only few studies have considered BTO thin films on a substrate, while it could be of great interest to take advantage of the surface properties of polarised films. Barbier and coworkers ${ }^{127}$ as well as Lu et al. ${ }^{28}$ have experimentally explored the polarisation of $\mathrm{BTO}$ thin films deposited on $\mathrm{Nb}: \mathrm{SrTiO}_{3}$ photo anodes. In the meantime, Pancotti et al. ${ }^{29]}$ have combined X-Ray Photoelectron Diffraction (XPD) and multiple scattering simulations to characterize the surface rumpling and relaxation of ferroelectric BTO(001) single crystal. Theoretically, very few studies have been dedicated to BTO thin films. Meyerheim et al. . $^{30 \mid 31}$ have considered $\mathrm{BaTiO}_{3}$ over either Fe, $\mathrm{Pt}$ or Pd(001) surfaces, but only in its paraelectric phase. Recently ${ }^{32}$ the out-of-plane polarisation of BTO has been carefully described for isolated BTO. However, this differs from reality because BTO thin films are rarely used alone 
but rather deposited on a conductive substrate such as Pt which offers good conductive properties as well as suitable epitaxy conditions. The substrate can broadly modify structural, electronic and polarisability properties of the overlayer. To take advantage as much as possible of ferroelectricity of BTO, it thus appears essential to thoroughly characterise its properties when grown over a realistic substrate.

Hence, in this paper, we present a detailed description of the nature of the $\mathrm{Pt}(001) / \mathrm{BTO}(001)$ interface. Then, we report a thorough analysis of the geometric and electronic influence of Pt substrate, as well as the influence of the thickness and the nature of the termination of the overlayers on the BTO polarisation.

\section{Computational details}

Density Functional Theory (DFT) calculations were performed using the Vienna $a b$ initio simulation package VASP code 33 34. The calculations were carried out with a plane-wave energy cutoff of $500 \mathrm{eV}$. The projector augmented wave (PAW) potentials were used to describe the electron-ion interaction $35 \mid 36$, which

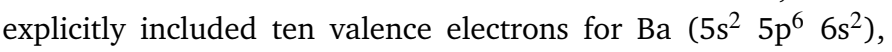
six for $\mathrm{O}\left(2 \mathrm{~s}^{2} 2 \mathrm{p}^{4}\right)$, twelve for Ti $\left(3 s^{2} 3 \mathrm{p}^{6} 4 \mathrm{~s}^{2} 3 \mathrm{~d}^{2}\right)$ and ten for Pt $\left(5 d^{9} 6 s^{1}\right)$. The generalised gradient approximation (GGA) was employed with Perdew-Burke-Ernzerhof (PBE) ${ }^{37}$ exchange correlation functional. As it is well known that DFT has issues to properly describe the strong correlation between $d$ electrons, the DFT $+\mathrm{U}$ approach was used by including an on-site Coulomb repulsion U-term for the Ti 3d electrons, adopting the Dudarev method 38 . The effective parameter $\mathrm{U}_{e f f}=3.5 \mathrm{eV}$ was applied and allowed to increase the band gap to $2.23 \mathrm{eV}$, getting closer to the experimental value $(3.2 \mathrm{eV}) \underline{39}$. This also allows to avoid spurious charge transfer between the substrate and the overlayer; results obtained in this way compare well with previous study. $\underline{40}$

The convergence threshold on total energy was set to $10^{-6}$ $\mathrm{eV}$. The structural optimisation was stopped when the forces converged below $0.01 \mathrm{eV} / \AA$. In the following, different periodicities of unit cells have been considered; a $7 \times 7 \times 1$ Monkhorst-Pack 41 $k$-point mesh was used to sample the first Brillouin zone for the smallest periodicity. For larger cells, the grid was adapted accordingly.

Prior to any surface calculation, tetragonal bulk BTO was modeled. The lattice parameters of the stable structure was found to be $a=4.006 \AA$ and $c=4.184 \AA$ corresponding to a $c / a$ ratio of 1.044. These values are in pretty good agreement with other DFT studies 42 where $a$ has been calculated between 3.954 and $4.013 \AA$ for a $c / a$ ratio between 1.006 and 1.045 , depending on the level of calculations. This also fits with experiments which have measured $a=3.991 \AA, c=4.036 \AA$ and thus $c / a=1.011$ at room temperature. ${ }^{18}$ Following the Berry phase method a spontaneous polarisation of $P=31.7 \mu \mathrm{C} . \mathrm{cm}^{-2}$ was calculated. This value stands in the range (from 24.3 to $35.1 \mu \mathrm{C} . \mathrm{cm}^{-2}$ ) of previously

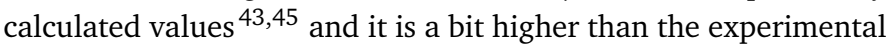
value of $27 \mu \mathrm{C} . \mathrm{cm}^{-2} 47 / 48$. However this is consistent with the well-known fact that GGA tends to overestimate the tetragonality of BTO and thus its polarisation.

The Pt(001) substrate was modelled by a five layers slab, in which

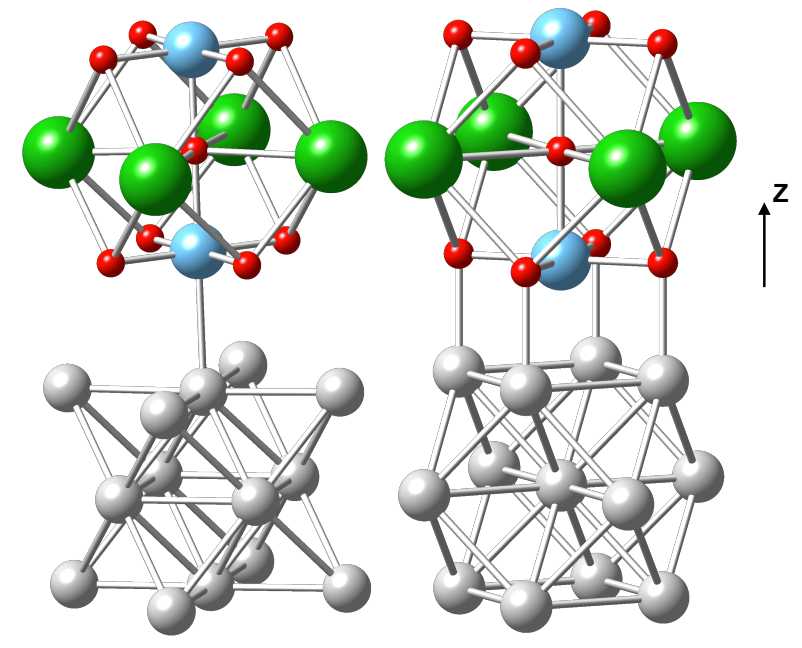

Fig. 1 Side view of the top (left) and hollow (right) adsorption positions of $\mathrm{BaTiO}_{3}$ on $\mathrm{Pt}(001)$ in the case of the $\mathrm{Pt} / \mathrm{TiO}_{2}$ interface. The $z$ axis stands along the [001] direction. For sake of clarity only 3 layers are reported for both the substrate and the overlayer. $\mathrm{Pt}$ atoms are reported in gray, $\mathrm{Ba}$ in green, $\mathrm{Ti}$ in blue and $\mathrm{O}$ in red.

the bottom two layers were fixed at their bulk positions. To reproduce as faithfully as possible the experimental strain induced by a platinum substrate on a BTO overlayer, Pt was described with its experimental lattice constant, namely $a_{P t}=3.92 \AA$, inducing a $2.1 \%$ lattice mismatch for epitaxially grown BTO. Influence of thickness was considered through BTO slabs from 7 to 15 layers, while influence of width, through slabs of different periodicities. In each case, all atoms of BTO slabs, either isolated or deposited, were allowed to relax. A $20 \AA$ vacuum was used to separate periodic images along the $\mathrm{z}$ direction.

\section{Results}

\subsection{The Pt/BaTiO 3 (001) interface}

Before studying the polarisation of the BTO thin films, it is mandatory to properly describe the $\mathrm{Pt}(001) / \mathrm{BaTiO}_{3}(001)$ interface. Several combinations have to be taken into account. First both terminations of BTO, namely $\mathrm{TiO}_{2}$ and $\mathrm{BaO}$, have to be considered for each interface: the Pt/BTO one and the BTO/vacuum. At this stage, an additional parameter occurs: the Pt/BTO alignment for the growth of the first layer. In the following "Top" and "Hollow" will refer to the position of the Ti (resp. Ba) atoms of the BTO first layer regarding to the Pt surface, as reported on Figure 1 for the $\mathrm{Pt} / \mathrm{TiO}_{2}$ interface. To analyse these interfaces, we have considered the adhesion energy defined as: $\mathrm{E}_{a d h}=\mathrm{E}_{(P t / B T O)}$ $\mathrm{E}_{(P t)}-\mathrm{E}_{(B T O)}$. This energy was decomposed into two components through the following equation: $\mathrm{E}_{a d h}=\mathrm{E}_{\text {int }}+\mathrm{E}_{\text {strain }}$. The former is negative and corresponds to the interaction between BTO and $\mathrm{Pt}$, while the latter is positive and corresponds to the strain induced in BTO by the epitaxy. All numerical results are summarised in Table 1

First of all, $\mathrm{Pt} / \mathrm{TiO}_{2}$ and $\mathrm{Pt} / \mathrm{BaO}$ interfaces behave quite differently. While hollow and top position can barely be distinguished when growth starts through $\mathrm{BaO}$ surface, the hollow site is clearly 


\begin{tabular}{c|c||c|c||c|c}
\hline \multicolumn{1}{c||}{} & \multicolumn{4}{c}{5 layers $\mathrm{Pt}$} \\
\hline \multicolumn{2}{c||}{ on Pt } & \multicolumn{2}{c|}{$\mathrm{TiO}_{2}$} & \multicolumn{2}{c}{$\mathrm{BaO}$} \\
\hline \multicolumn{2}{c||}{ upper termination } & $\mathrm{TiO}_{2}$ & $\mathrm{BaO}$ & $\mathrm{TiO}_{2}$ & $\mathrm{BaO}$ \\
\hline \multicolumn{2}{c||}{$\mathrm{E}_{\text {strain }}$} & 30 & 32 & 32 & 43 \\
\hline \multirow{2}{*}{ Top } & $\mathrm{E}_{\text {adh }}$ & 12 & 17 & -15 & 12 \\
& $\mathrm{E}_{\text {int }}$ & -19 & -15 & -47 & -32 \\
& $\mathrm{~d}_{P t / c a t}$ & 2.79 & 2.84 & 3.37 & 3.35 \\
\hline \multirow{2}{*}{ Hollow } & $\mathrm{E}_{\text {adh }}$ & -31 & -30 & -17 & 10 \\
& $\mathrm{E}_{\text {int }}$ & -62 & -62 & -50 & -33 \\
& $\mathrm{~d}_{P t / \text { cat }}$ & 2.19 & 2.20 & 3.77 & 3.78
\end{tabular}

Table 1 Adhesion energies, $\mathrm{E}_{\text {adh }}$ (in meV. $\AA^{-2}$ ), interaction energies, $\mathrm{E}_{\text {int }}$ (in meV. $\AA^{-2}$ ), and distances, $d_{P t / c a t}$ (in $\AA$ ), between Pt from the substrate and cation (Ba or Ti) from the deposit, for an overlayer of BTO deposited on a $\mathrm{Pt}(001)$ slab. For symmetric overlayers, namely $\left[\mathrm{TiO}_{2} / \mathrm{TiO}_{2}\right]$ (or $[\mathrm{BaO} / \mathrm{BaO}])$, a BTO thin film of 11 layers is used, while for asymmetric ones, namely $\left[\mathrm{TiO}_{2} / \mathrm{BaO}\right]$ (or $\left[\mathrm{BaO} / \mathrm{TiO}_{2}\right]$ ), the films present a 10 layers thickness. Top and hollow refer to the position of the first layer cations (Ba or Ti) towards platinum.

favoured when $\mathrm{TiO}_{2}$ is in contact with platinum. The similarity between top and hollow sites for the $\mathrm{Pt} / \mathrm{BaO}$ interface is directly related to the weak $\mathrm{Pt} / \mathrm{Ba}$ interaction, leading to a large distance between the two surfaces. As reported in Table 1 , the distance between platinum and baryum is more than $3 \AA$ : 3.4 and $3.8 \AA$ for top and hollow sites, respectively. This wide gap between both interfaces neutralises the effects related to different adsorption sites. This is confirmed by the fact that the substrate/layer interaction $\left(\mathrm{E}_{\text {int }}\right)$ is identical for both positions. Hence, when growth starts from the $\mathrm{BaO}$ termination, the only difference comes from the upper termination. In fact, the symmetric [BaO/BaO] overlayers are slightly unstable $\left(\mathrm{E}_{a d h} \approx 15 \mathrm{meV} / \AA^{2}\right)$ while asymmetric $\left[\mathrm{BaO} / \mathrm{TiO}_{2}\right]$ ones present a small stabilisation $\left(\mathrm{E}_{a d h} \approx-\right.$ $15 \mathrm{meV} / \AA^{2}$ ). This disparity can be analysed through the nature (symmetric or asymmetric) of the overlayer, leading to different sensitivities to interlayers relaxations induced by the presence of the substrate only on one side.

On the contrary, for the $\mathrm{Pt} / \mathrm{TiO}_{2}$ interface, top and hollow positions can be easily distinguished: the hollow adsorption is favoured, while the top one is unstable. This comes mainly from the strain induced over BTO by the substrate. When it grows over platinum, BTO endures a $2.1 \%$ geometrical constraint due to the difference of lattice parameter with platinum. This strain leads to an energy cost around $30 \mathrm{meV} / \AA^{2}$ for both $\left[\mathrm{TiO}_{2} / \mathrm{TiO}_{2}\right]$ and $\left[\mathrm{TiO}_{2} / \mathrm{BaO}\right] \mathrm{BaTiO}_{3}$ slabs. Hence, even if the $\mathrm{Pt} / \mathrm{TiO}_{2}$ interface interaction energies are always negative, the stabilisation observed for top position is not large enough to overcome the strain cost. As a consequence, the final adhesion energy is positive leading to an unfavored growth over top positions. These results are consistent with previous studies. In particular Rappe and coworkers 26 also found that the $\mathrm{Pt} / \mathrm{TiO}_{2}$ interface with $\mathrm{Ti}$ atoms in hollow sites is the most stable. As shown by Stengel et al. $\stackrel{20}{ }$, this is mainly related to the nature of the Pt-O bond. Indeed, when Ti atoms are located on hollow sites, all surrounding oxygens are on top of $\mathrm{Pt}$ atoms (see Figure 1), leading to a strong interaction.

According to results reported above, in the following we will only focus on the $\mathrm{Pt} / \mathrm{TiO}_{2}$ interface, with $\mathrm{Ti}$ atoms on hollow sites.

\subsection{Stability of ferroelectric phases}

Up to now only one theoretical study 32 has considered the out-of-plane polarisation of BTO not involved in capacitors. This lack of studies, despite numerous potential applications, is certainly due to the strong limitations induced by the modelling of such a system. In fact thin films of ferroelectrics, accumulate charges at their surface. This leads to an electric field (called depolarising field) which tends to counter this accumulation to put the system back to neutral. Hence to keep the out-of-plane polarisation, it is mandatory to use a computational protocol that avoids this depolarisation. Different methods, $42,49-51$ all presenting pros and cons, have already been proposed. In the following we will apply the method firstly introduced by Migoni et al. $\frac{52}{52}$ for a molecular dynamics study of deposited $\mathrm{PbTiO}_{3}$ thin films and then developed by Shimada ${ }^{51}$ for periodic DFT study of free-standing $\mathrm{PbTiO}_{3}$ thin films. This method consists in dividing the thin films into two domains of opposite polarisation, in order to obtain a simulation cell without any net dipole moment, thus preventing all depolarisation issues. One non negligible advantage of this method is that beyond the calculation trick, this also depicts the reality. In fact the existence of polydomains has already been evidenced experimentally for such ferroelectrics 53 . 55 .

In the case of BTO deposited on platinum, the metallic character of the substrate allows a charge accumulation which compensates the surface charge of the ferroelectric 19 , and thus simplifies issues relative to depolarisation. However, this accumulation at the bottom interface can not be infinite - this would lead to a divergence of the system -, thus the use of the opposite domains' model ${ }^{51 / 52}$ is still necessary to create some alternation of charge accumulation and depletion in the top layers of the substrate, leading the remaining of platinum unaffected. Besides, the thickness of platinum substrate required for surface dipole convergence has also been checked. Contrary to what happens in capacitors 56 , five layers of platinum are sufficient for overlayers.

First of all, we have determined the BTO critical thickness required to favour the ferroelectric phase. To do so, symmetric $\left[\mathrm{TiO}_{2} / \mathrm{TiO}_{2}\right]$ BTO systems from 7 to 15 layers have been considered, either isolated or deposited on Pt substrate. We first discuss only the two unit cells periodicity. To analyse the electronic influence of the substrate on the polarisation, BTO deposited on platinum is compared with isolated BTO already constrained at the Pt lattice parameter, namely $3.92 \AA$. The relative stability of ferro- and paraelectric phases is compared for all these cases and reported on Figure 2. According to these results the stability of the ferroelectric phase over the paraelectric one increases as the thickness increases, especially over platinum. Nevertheless, the presence of platinum has no influence on the critical thickness that stabilises polarised systems: in both cases symmetric ferroelectric systems are stable for slabs of 11 or more layers, namely $23 \AA$.

Critical thickness has also been determined for the four and six unit cells periodicity. While it remains unchanged for isolated BTO (see Figure 2), in presence of platinum ferroelectric phase 


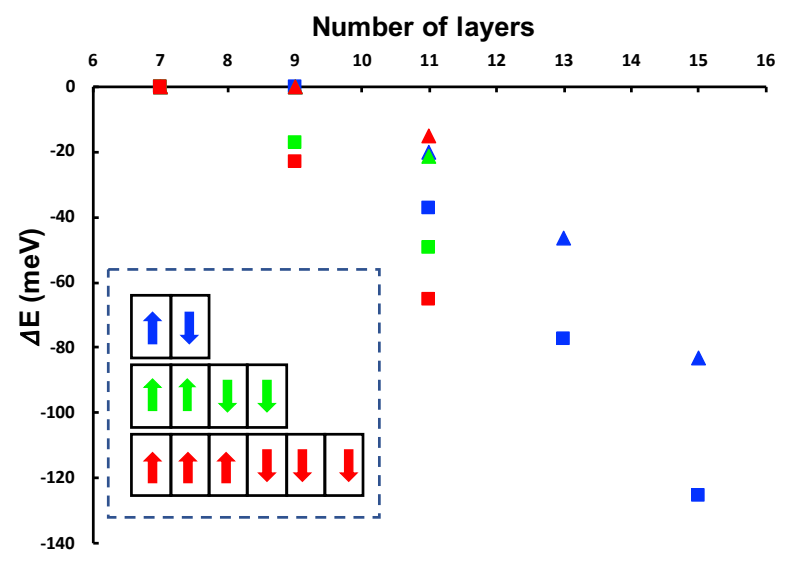

Fig. 2 Relative stability between ferroelectric and paraelectric phases: $\Delta \mathrm{E}=\left(\mathrm{E}_{\text {ferro }}-\mathrm{E}_{\text {para }}\right) /(\mathrm{N} / 2)$ in meV, as a function of the number of layers and of the number of lateral unit cells $(\mathrm{N})$. Triangles correspond to isolated strained BTO, squares to BTO deposited on Pt. Periodicities of two $(\mathrm{N}=2)$, four $(\mathrm{N}=4)$ and six $(\mathrm{N}=6)$ unit cells are reported in blue, green and red, respectively. For sake of clarity, these three periodicities are sketched in the insert.

is stabilised from 9 layers, demonstrating a different behaviour between free-standing and deposited BTO. This result confirms the role of boundary conditions, already evidenced both theoretically $\sqrt{52}$ and experimentally $\sqrt{57}$, on the critical thickness. The influence of lateral periodicity has been carefully analysed for the 11 layers films, with and without platinum. For the later case, our findings are fully consistent with previous results

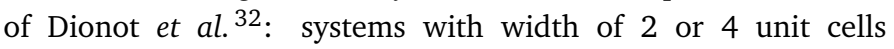
are the most stable ( $\Delta \mathrm{E}$ around $20 \mathrm{meV}$ ), while those with 6 unit cells of periodicity are not favoured with $\Delta \mathrm{E}=-15 \mathrm{meV}$. This behaviour is substantially modified by the presence of platinum. In fact, the larger stabilisation of the ferroelectric phase over the paraelectric already observed for the 2 unit cells system, is still present, but is even larger as the periodicity increases. Hence for BTO deposited on Pt, the six unit cells systems (sketched in red in the insert of Figure 2) is clearly favoured.

\subsection{Polarisation patterns}

Starting from the 11 layers thin films, the local polarisation has been analysed in details. To do so, the polarisation of each unit cell centered on an oxygen atom of a $\mathrm{BaO}$ layer (delimited with lines on Figure 3) has been calculated with the formula developed by Vanderbilt $\frac{58}{1}$ and already used by Shimada 51 :

$$
\mathbf{P}=\frac{e}{\Omega_{c}} \sum_{j} \omega_{j} \mathbf{Z}_{j}^{*} \mathbf{u}_{j},
$$

where $e$ and $\Omega_{c}$ refer to the electron charge and the volume of the unit cell, respectively. The index $j$ of the sum covers all atoms in the unit cell, with $\omega_{j}$ and $\mathbf{u}_{j}$, the weight of atom $j$ in the unit cell and its atomic displacement vector from the ideal lattice position, respectively. Finally, $\mathbf{Z}_{j}^{*}$ is the Born effective charge tensor of atom $j$ in the cubic bulk of $\mathrm{BaTiO}_{3}$.

The local polarisations are reported for each unit cell on
Figure 3 for both isolated strained and deposited BTO. The left side corresponds to the $P_{u p}$ polarisation, with displacements of Ti atoms outwards, while the right side corresponds to the $P_{\text {down }}$ with displacements of Ti atoms inwards. Reported values correspond only to the contribution along the $\mathrm{Z}$ axis. In fact for the two unit cells periodicity, lateral contributions are null. We first focus on isolated strained BTO. While the increase of the thickness favours the ferroelectric phase towards the paraelectric one, there is no crucial change in polarisation patterns. A low polarisation (between 5 and $12 \mu \mathrm{C} . \mathrm{cm}^{-2}$ ) is always observed at the surface, while polarisation of central unit cells increases as the thickness increases, to get closer to the strained bulk polarisation. In fact when a $2.1 \%$ compressive strain is applied to the BTO bulk, its polarisation increases from 31.7 to $37.1 \mu \mathrm{C} . \mathrm{cm}^{-2}$. This corresponds to a gain of almost $20 \%$ of polarisation, in agreement with previous results: Junquera and Ghosez ${ }^{19}$ have observed a $29 \%$ increase for BTO involved in capacitors with the same kind of constraint. Qualitatively, this behaviour fully agrees with the previous study of Dionot et al. ${ }^{32}$, for a $3.8 \%$ constraint. The electronic influence of platinum on the polarisation is now considered for the two unit cells periodicity. First of all, despite the use of symmetric initial configurations, optimised ferroelectric BTO over Pt, presents clear dissymmetries of local polarisation (see Figure 3). If this loss of symmetry has to be analysed, it is worth mentioning that it has no consequence such as the appearance of a depolarising field as mentioned above. Indeed, the presence of a metallic substrate allows accumulation of charges which compensate the ferroelectric surface charge density 19 . Three parts can thus be distinguished in the overlayers. First of all the upper part, namely the surface unit cells close to vacuum, presents a polarisation similar to the one observed without Pt. Absolute values between 5 and $15 \mu \mathrm{C} . \mathrm{cm}^{-2}$ are calculated with opposite polarisation for neighbouring cells. For central unit cells, we also observed a behaviour similar to the one of isolated BTO. The local polarisation increases in comparison to the surface values. One can mention that in presence of Pt, polarisation is slightly higher and thus closer to the bulk value. Finally, contrary to other parts of the slab, a strong difference of behaviour occurs at the Pt/BTO interface. The symmetry is broken and neighbouring cells now present a local polarisation with identical orientation, even if the order of magnitude completely differs. This phenomenon can be explained through the bonding of interfacial atoms which can strongly modify the local ferroelectricity, as already evidenced for $\mathrm{KNbO}_{3}$ capacitors by Tsymbal and coworkers ${ }^{23}$. According to this study, the bonds created between the substrate and the perovskite impose some movement restrictions for atoms at the boundaries, inducing a local polarisation at the interface even for the paraelectric capacitors. In agreement with these results, we have analysed the displacements of the first layer atoms in the paraelectric phase for our system: the Ti atoms of the first layer are pushed $0.06 \AA$ towards the $\mathrm{O}$ atoms in the presence of platinum. This phenomenon is maintained in the ferroelectric phase increasing the $\mathrm{P}_{\text {down }}$ polarisation and decreasing the $\mathrm{P}_{u p}$ one, leading to neighbouring cells with polarisation in the same direction. To conclude the bonding of the ferroelectric BTO 
over the substrate induces some disruptions on the polarisation in the first deposited layers, and thus will modify the global polarisation: in presence of platinum, the global $P_{u p}$ is lowered, while $\mathrm{P}_{\text {down }}$ is increased. Another important conclusion concerns the independency of the global polarisation behaviour with the slab thickness. In fact, in both BTO and Pt/BTO cases, as soon as the ferroelectric state becomes stable, the distribution of polarisation along the layers is identical whatever the total size of the slab.

The influence of lateral periodicity is now discussed for the 11 layers thickness. Results for the three considered periodicities, namely 2, 4 and 6 unit cells, are reported on Fig 4 . From this graphic, two main conclusions can be drawn. First of all, whatever the periodicity, the platinum has always the same influence on the polarisation. In the three cases we observe the same patterns as previously described for the two cells periodicity, namely a large asymmetry at the Pt/BTO interface, then a polarisation close to the bulk one in the middle cells, finally an opposite polarisation for the neighbouring upper cells. Secondly, contrary to the smallest width, higher periodicities (like 4 and 6 unit cells) allow the appearance of closure domains similar to the ones already observed by Shimada et al. for $\mathrm{PbTiO}_{3}$ 51. Again, except at the Pt/BTO interface, the presence of the substrate does not have a large influence on the patterns, for a given periodicity. However, the main difference will come from the observed periodicity. As reported on Figure 2, only the 2 or 4 unit cells periodicities are observed for isolated BTO, while in presence of platinum, larger periodicities are favoured, allowing larger domains. As reported on Fig 4 the increase of lateral periodicity leads also to an increase of local polarisation. Hence in presence of platinum, larger domains with higher polarisation are obtained.

\subsection{Influence of upper termination}

As mentioned above, as soon as the growth starts from $\mathrm{TiO}_{2}$ interface with Ti in hollow site, the top termination has no influence on the stability. Hence, one can imagine that the film can be terminated either by a $\mathrm{BaO}$ or a $\mathrm{TiO}_{2}$ layer, depending on experimental conditions. It is thus important to study if the modification of the termination has some influence on the intensity of the polarisation. The $\left[\mathrm{TiO}_{2} / \mathrm{BaO}\right]$ slab either isolated or deposited on $\mathrm{Pt}$ is thus compared to the reference $\left[\mathrm{TiO}_{2} / \mathrm{TiO}_{2}\right]$ slab. To allow a thorough comparison, the isolated strained $[\mathrm{BaO} / \mathrm{BaO}]$ slab is also considered. In the case of $\mathrm{BaO}$ termination, the local polarisation is calculated in a unit cell centered on a Ti atom from a $\mathrm{TiO}_{2}$ plan. For the $\left[\mathrm{TiO}_{2} / \mathrm{BaO}\right]$ systems, the asymmetry of the slab prevents the decomposition of the slab in an integer number of unit cells. As we primary focus on the polarisation at the vacuum / slab interfaces, for the asymmetric systems we start the decomposition from the top $\mathrm{BaO}$ surface and we do not calculate the local polarisation of the last layer, as reported on Figure 5. As a consequence, the local polarisation is calculated for unit cells centered on Ti atoms as in the case of $[\mathrm{BaO} / \mathrm{BaO}]$ systems.

As for symmetric slabs, the isolated strained systems are first considered. In Figure 6 the local polarisation for the $\mathrm{P}_{u p}$ and

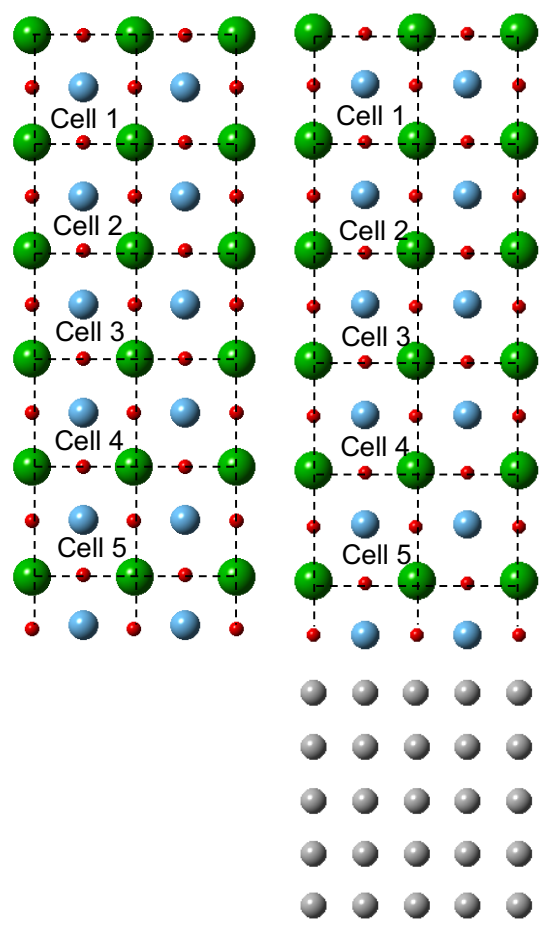

Fig. 5 Paraelectric 12 layer asymmetric $\left[\mathrm{BaO} / \mathrm{TiO}_{2}\right]$ slab either isolated and strained on the left or deposited on platinum on the right. Ba atoms are represented in green, $\mathrm{Ti}$ in blue, $\mathrm{O}$ in red and $\mathrm{Pt}$ in gray. The delimitation as well as the numbering of the cells used for the discussion are also reported.

the $\mathrm{P}_{\text {down }}$ sides is reported for the first four surface cells (Cells 1 to 4 in Figure 5) of the 11 layers $\left[\mathrm{TiO}_{2} / \mathrm{TiO}_{2}\right]$ and $[\mathrm{BaO} / \mathrm{BaO}]$ slabs and for the 12 layers $\left[\mathrm{BaO} / \mathrm{TiO}_{2}\right]$. The polarisation of the symmetric $[\mathrm{BaO} / \mathrm{BaO}]$ slab is clearly the highest. This is directly related to the chemical nature of termination. In fact, in the case of $\mathrm{BaO}$ terminated slabs, the unit cells recover the original nature of bulk BTO unit cells. The natural movement of Ti atoms is thus favoured, allowing a better amplitude of displacements. The increase of polarisation is especially true at the surface of the $\mathrm{P}_{\text {down }}$ side and in the center part of the slab. For $[\mathrm{BaO} / \mathrm{BaO}]$ system, the polarisation of the strained bulk is almost reached for cell 3: $\mathrm{P}_{\text {up }}\left(\right.$ Cell 3) $=\mid \mathrm{P}_{\text {down }}\left(\right.$ Cell 3) $\mid=32 \mu \mathrm{C} . \mathrm{cm}^{-2}\left(\mathrm{P}_{\text {strained }}\right.$ (bulk) $=37$ $\left.\mu \mathrm{C} . \mathrm{cm}^{-2}\right)$. These results clearly show that the $[\mathrm{BaO} / \mathrm{BaO}]$ system has a higher polarisation than the $\left[\mathrm{TiO}_{2} / \mathrm{TiO}_{2}\right]$ ones. This interesting behaviour is partially kept for asymmetric systems. In fact, as observed on Figure 6 , the $\left[\mathrm{BaO} / \mathrm{TiO}_{2}\right]$ system presents an intermediate polarisation between $\left[\mathrm{TiO}_{2} / \mathrm{TiO}_{2}\right]$ and $[\mathrm{BaO} / \mathrm{BaO}]$. Again, the polarisation of middle cells is increased compared to symmetric $\mathrm{TiO}_{2}$ systems, as well as the surface polarisation of the $\mathrm{P}_{\text {down }}$ side.

Finally, the local polarisation of $\left[\mathrm{BaO} / \mathrm{TiO}_{2}\right]$ is now evaluated when it is deposited on Pt. Results are reported on Figure 7 According to this graph, the presence of platinum slightly increases again the value of local polarisation, as found for the $\left[\mathrm{TiO}_{2} / \mathrm{TiO}_{2}\right]$ system. Hence, using appropriate experimental con- 


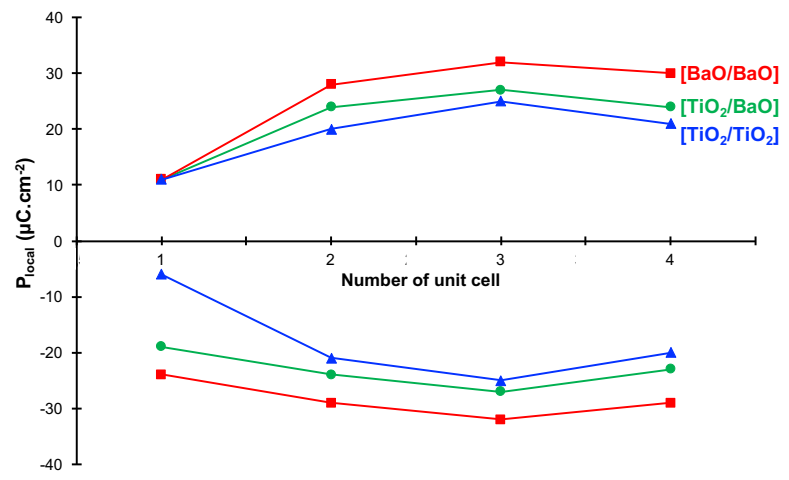

Fig. 6 Evolution of local polarisation (in $\mu \mathrm{C} . \mathrm{cm}^{-2}$ ) in the symmetric slabs of 11 layers with either two $\mathrm{TiO}_{2}$ terminations (blue curves with triangles) or two $\mathrm{BaO}$ terminations (red curves with squares) and in asymmetric $\left[\mathrm{TiO}_{2} / \mathrm{BaO}\right.$ ] slabs of 12 layers (green curves with circles). Only values for the four first cells starting from the $\mathrm{BaO}$ surface in the case of asymmetric slabs are reported. Positive values refer to the $\mathrm{P}_{u p}$ side, negative to the $\mathrm{P}_{\text {down }}$ one.

ditions that lead to an upper $\mathrm{BaO}$ termination should allow a higher polarisation and thus modify the properties of the material.

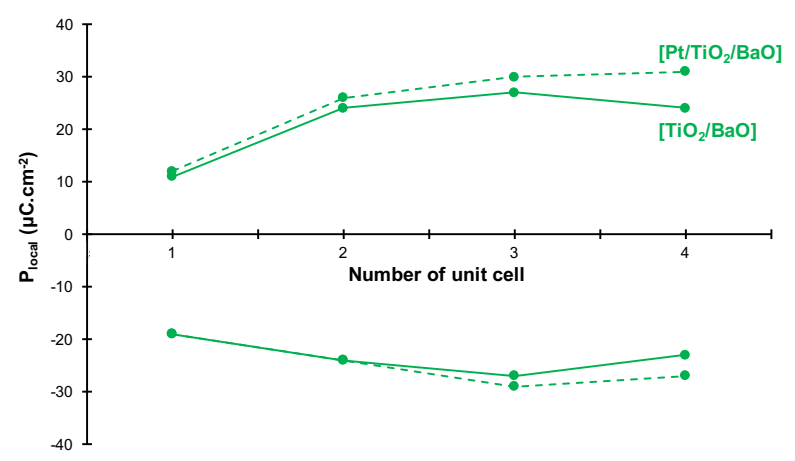

Fig. 7 Evolution of local polarisation (in $\mu \mathrm{C} . \mathrm{cm}^{-2}$ ) in asymmetric $\mathrm{TiO}_{2} / \mathrm{BaO}$ slabs of 12 layers either isolated (plain green curve) or deposited on Pt (dotted green curve) Only values for the four first cells starting from the $\mathrm{BaO}$ interface with vacuum are reported. Positive values refer to the $\mathrm{P}_{u p}$ side, negative to the $\mathrm{P}_{\text {down }}$ one

\section{Conclusions}

In summary, we have performed a thorough analysis of the ferroelectric behaviour of $\mathrm{BaTiO}_{3}(001)$ slabs epitaxially grown on $\mathrm{Pt}(001)$. The paraelectric BTO was firstly considered to determine the nature of the Pt/BTO interface. We thus showed that the growth always starts with the $\mathrm{TiO}_{2}$ termination with titanium atoms in hollow positions, and oxygen atoms on top of platinum. Under the $2.1 \%$ compressive strain induced by the lattice mismatch between the substrate and the overlayer, ferroelectric slabs of BTO are stabilised over paraelectric ones for thicknesses larger than $23 \AA$ (11 layers). Beyond this thickness, all symmetric $\left[\mathrm{TiO}_{2} / \mathrm{TiO}_{2}\right]$ slabs present the same evolution of polarisation: local polarisation at the surface is lower than the one calculated inside the slab, which tends to the strained bulk polarisation. The presence of a platinum substrate induces several modifica- tions on the BTO overlayers. On an energetic point of view, platinum has two consequences. First of all, it increases the stability of ferroelectric slabs over paraelectric ones, and lowers the thickness for stabilising the ferroelectric phase. In presence of platinum, ferroelectric phase is observed from 9 layers for domains of width of two unit cells or more. Secondly, the presence of a substrate modifies the size of the domains. While for isolated BTO slabs, the small two or four unit cells periodicities are favoured over larger ones, in presence of platinum, larger domains will be observed. Concerning the polarisation, at a given periodicity, both deposited and isolated BTO slabs present a similar qualitative behaviour. From a quantitative point of view, three differences have to be mentioned: the stability of ferroelectric slabs over paraelectric ones is increased in the presence of platinum. Secondly, the local polarisation is modified at the Pt/BTO interface because of Pt-O bonding. Thirdly, the local polarisation of the central unit cells is increased in the presence of platinum. Finally an important change due to the presence of platinum, is the kind of observed domains. As larger cells are favoured for overlayers, larger domains with clearer closure walls will be observed.

Finally the influence of the upper termination on the polarisation was also analysed through the study of asymmetric $\left[\mathrm{TiO}_{2} / \mathrm{BaO}\right]$ slabs: the presence of an upper $\mathrm{BaO}$ termination induces an increase of the local polarisation of the middle cells, but also of the surface polarisation of the $\mathrm{P}_{\text {down }}$ side.

\section{Acknowledgements}

Calculations were performed using HPC resources from DNUM CCUB (Centre de Calcul de l'Université de Bourgogne). The authors also thank the ANR for financial support through project ANR-15-CE05-PHOTO-POT.

\section{Notes and references}

1 M. Rioult, S. Datta, D. Stanescu, S. Stanescu, R. Belkhou, F. Maccherozzi, H. Magnan and A. Barbier, Appl. Phys. Lett., 2015, 107, 103901.

2 S. Park, C. W. Lee, M.-G. Kang, S. Kim, H. J. Kim, J. E. Kwon, S. Y. Park, C.-Y. Kang, K. S. Hong and K. T. Nam, Phys. Chem. Chem. Phys., 2014, 16, 10408-10413.

3 W. Ji, K. Yao, Y. F. Lim, Y. C. Liang and A. Suwardi, Appl. Phys. Lett., 2013, 103, 062901.

4 Y. Inoue, K. Sato and H. Miyama, J. Phys., 1986, 90, 28092810.

5 D. Tiwari and S. Dunn, J. Mater. Sci., 2009, 44, 5063-5079.

6 L. Li, P. A. Salvador and G. S. Rohrer, Nanoscale, 2014, 6, 24-42.

7 A. Kakekhani, S. Ismail-Beigi and E. I. Altman, Surf. Sci., 2016, 650, 302-316.

8 M. Qin, K. Yao and Y. C. Liang, Appl. Phys. Lett., 2008, 93, 122904.

9 I. Grinberg, D. V. West, M. Torres, G. Gou, D. M. Stein, L. Wu, G. Chen, E. M. Gallo, A. R. Akbashev, P. K. Davies, J. E. Spanier and A. M. Rappe, Nature, 2013, 503, 509-512.

10 Y. Yuan, Z. Xiao, B. Yang and J. Huang, J. Mater. Chem. A, 2014, 2, 6027-6041. 
11 J. L. Giocondi and G. S. Rohrer, Chem. Mater, 2001, 13, 241242.

12 J. L. Giocondi and G. S. Rohrer, J. Phys. Chem. B, 2001, 105, 8275-8277.

13 N. V. Burbure, P. A. Salvador and G. S. Rohrer, J. Am. Ceram. Soc., 2006, 89, 2943-2945.

14 N. V. Burbure, P. A. Salvador and G. S. Rohrer, Chem. Mater., 2010, 22, 5831-5837.

15 J. W. Edwards, R. Speiser and H. L. Johnston, J. Am. Chem. Soc., 1951, 73, 2934-2935.

16 B. C. Frazer, H. R. Danner and R. Pepinsky, Phys. Rev., 1955, 100, 745-746.

17 J. Harada, T. Pedersen and Z. Barnea, Acta Crystallogr. Sect. A, 1970, 26, 336-344.

18 G. H. Kwei, A. C. Lawson, S. J. Billinge and S. W. Cheong, J. Phys. Chem., 1993, 97, 2368-2377.

19 J. Junquera and P. Ghosez, Nature, 2003, 422, 506.

20 M. Stengel, D. Vanderbilt and N. A. Spaldin, Nat. Mater., 2009, 8, 392.

21 M. Stengel, D. Vanderbilt and N. A. Spaldin, Phys. Rev. B, 2009, 80, 224110.

22 Y. Umeno, B. Meyer, C. Elsässer and P. Gumbsch, Phys. Rev. B, 2006, 74, 060101(R).

23 C. Duan, R. Sabirianov, W. Mei, S. Jaswal and E. Tsymbal, Nano Lett., 2006, 6, 483.

24 P. Aguado-Puente and J. Junquera, Phys. Rev. Lett., 2008, 100, 177601.

25 Y. Umeno, J. Albina, B. Meyer and C. Elsässer, Phys. Rev. B, 2009, 80, 205122.

26 N. Sai, A. Kolpakd and A. Rappe, Phys. Rev. B, 2005, 72, 020101(R).

27 S. Datta, M. Rioult, D. Stanescu, H. Magnan and A. Barbier, Thin Solid Films, 2016, 607, 7-13.

28 H. A. Lu, L. A. Wills and B. W. Wessels, Appl. Phys. Lett., 1994, 64, 2973-2975.

29 A. Pancotti, J. Wang, P. Chen, L. Tortech, C. M. Teodorescu, E. Frantzeskakis and N. Barrett, Phys. Rev. B, 2013, 87, 184116.

30 H. L. Meyerheim, F. Klimenta, A. Ernst, K. Mohseni, S. Ostanin, M. Fechner, S. Parihar, I. V. Maznichenko, I. Mertig and J. Kirschner, Phys. Rev. Lett., 2011, 106, 087203.

31 H. L. Meyerheim, A. Ernst, K. Mohseni, I. V. Maznichenko, J. Henk, S. Ostanin, N. Jedrecy, F. Klimenta, J. Zegenhagen, C. Schlueter, I. Mertig and J. Kirschner, Phys. Rev. Lett., 2013, 111, 105501.

32 J. Dionot, G. Geneste, C. Mathieu and N. Barrett, Phys. Rev. B, 2014, 90, 014107.

33 G. Kresse and J. Furthmüller, Phys. Rev. B, 1996, 54, 1116911186.

34 G. Kresse and J. Furthmüller, Comput. Mater. Sci., 1996, 6, 15-50.

35 P. E. Blöchl, Phys. Rev. B, 1994, 50, 17953-17979.

36 K. G. and D. Joubert, Phys. Rev. B, 1999, 59, 1758-1775.
37 J. P. Perdew, K. Burke and M. Ernzerhof, Phys. Rev. Lett., 1996, 77, 3865-3868.

38 S. Dudarev, G. Botton, S. Savrasov, C. Humphreys and S. A.P., Phys. Rev. B, 1998, 57, 1505-1509.

39 K. Suzuki and K. Kijima, Jpn. J. Appl. Phys., 2005, 44, 2081.

40 M. Stengel, P. Aguado-Puente, N. A. Spaldin and J. Junquera, Phys. Rev. B, 2011, 83, 235112.

41 J. D. Pack and H. J. Monkhorst, Phys. Rev. B, 1977, 16, 17481749.

42 M. Fechner, S. Ostanin and I. Mertig, Phys. Rev. B, 2008, 77, 094112.

43 N. Iles, K. Driss Khodja, A. Kellou and P. Aubert, Comput. Mater. Sci., 2014, 87, 123-128.

44 D. I. Bilc, R. Orlando, R. Shaltaf, G. M. Rignanese, J. Íñiguez and P. Ghosez, Phys. Rev. B, 2008, 77, 165107.

45 E. S. Goh, L. H. Ong, T. L. Yoon and K. H. Chew, Comput. Mater. Sci., 2016, 117, 306-314.

46 R. A. Evarestov and A. V. Bandura, J. Comput. Chem., 2012, 33, 1123-1130.

47 W. J. Merz, Phys. Rev., 1953, 91, 513-517.

48 H. H. Wieder, Phys. Rev., 1955, 99, 1161-1165.

49 Y. Mi, G. Geneste, J. Rault, C. Matthieu, A. Pancotti and N. Barrett, J. Phys.: Condens. Matter, 2012, 24, 275901.

50 B. Meyer and D. Vanderbilt, Phys. Rev. B, 2001, 63, 205426.

51 T. Shimada, S. Tomoda and T. Kitamura, Phys. Rev. B, 2010, 81, 144116.

52 M. Sepliarsky, M. G. Stachiotti and R. L. Migoni, Phys. Rev. Lett., 2006, 96, 137603.

53 S. K. Streiffer, J. A. Eastman, D. D. Fong, C. Thompson, A. Munkholm, M. V. R. Murty, O. Auciello, G. R. Bai and G. B. Stephenson, Phys. Rev. Lett., 2002, 89, 067601.

54 N. Spaldin, Science, 2004, 304, 1606.

55 D. Fong, G. B. Stephenson, S. Streiffer, J. Eastman, O. Auciello, P. Fuoss and C. Thompson, Science, 2004, 304, 1650.

56 A. M. Kolpak, N. Sai and A. M. Rappe, Phys. Rev. B, 2006, 74, 054112.

57 P. Gao, Z. Zhang, M. Li, R. Ishikawa, B. Feng, H. J. Liu, Y. L. Huang, N. Shibata, X. Ma, S. Chen, J. Zhang, K. Liu, E. G. Wang, D. Yu, L. Liao, Y. H. Chu and Y. Ikuhara, Nat. Com., 2017, 8, 15549.

58 W. Zhong, R. D. King-Smith and D. Vanderbilt, Phys. Rev. Lett., 1994, 72, 3618. 


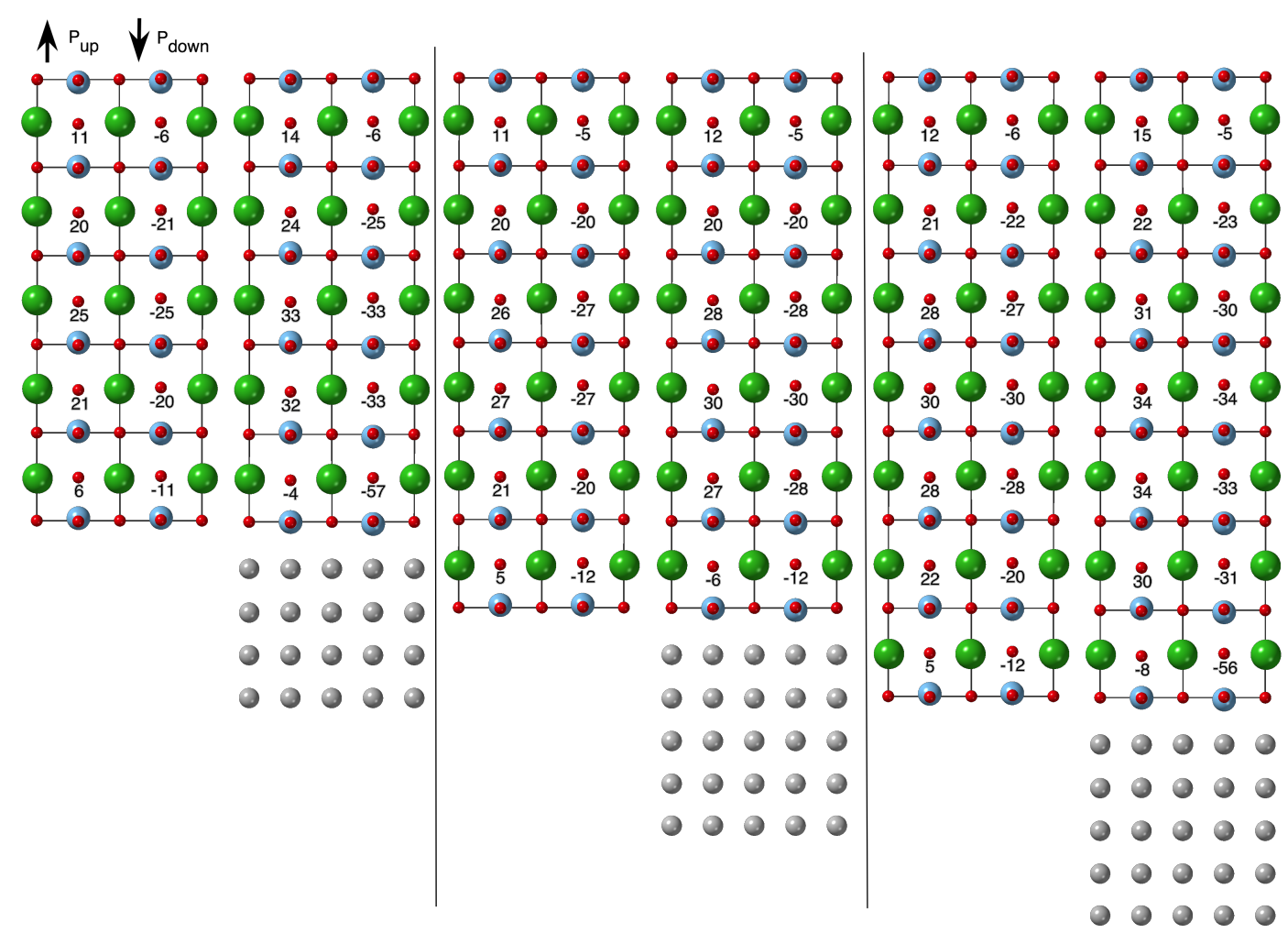

Fig. 3 Evolution of local polarisation as a function of BTO thickness for the two unit cells lateral periodicity. From left to right, slabs of 11 , 13 and 15 layers are considered. In each case, both isolated strained BTO and BTO deposited on Pt substrate are reported. Ba atoms are represented in green, $\mathrm{Ti}$ in blue, $\mathrm{O}$ in red and $\mathrm{Pt}$ in gray. Values of local polarisation in the out-of-plane direction is reported for each unit cell in $\mu \mathrm{C} . \mathrm{cm}^{-2}$.
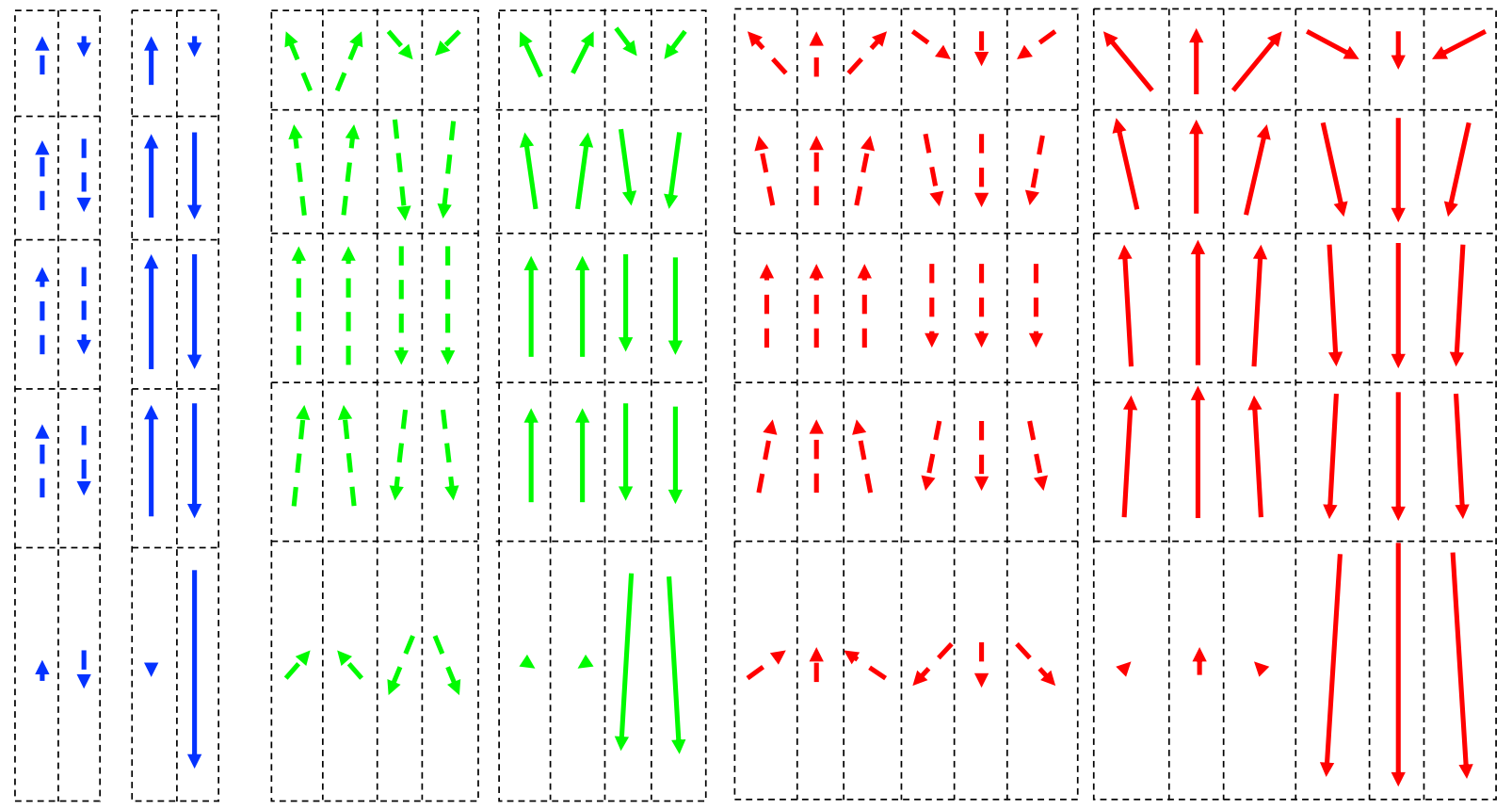

Fig. 4 Vectorial representation of local polarisation for two (in blue), four (in green) and six (in red) unit cells periodicities, for the 11 layers slab. Both isolated BTO (dotted arrows) and BTO deposited on Pt (plain lines arrows) are reported. Black dotted cells are guidelines. They represent, without respect of the scale, the unit cells centered on an oxygen atom of a $\mathrm{BaO}$ layer, used for the calculation of local polarisation. Cells in contact with $\mathrm{Pt}$ are the ones at the bottom of the pictures, while the interface with vacuum is at the top. 